\title{
Aplicação de Modelos Múltiplos na Determinação de Níveis de PREJUÍzo PARA A INTERAÇÃo Solanum americanum E TOMATE DE INDÚSTRIA ${ }^{1}$
}

\author{
Evaluation of Economic Levels for the Solanum americanum x Tomato Crop Interaction using \\ Multiple Models \\ PORTUGAL, J.M.2 e MOREIRA, I. ${ }^{3}$
}

\begin{abstract}
RESUMO - Este estudo apresenta um conceito unificador, que conjuga as teorias de densidade crítica e período crítico. Hipotetiza-se que não existe um valor fixo para a densidade crítica e para o período crítico, mas que os seus valores são interdependentes. A demonstração dessa hipótese é fundamentada em estudos de interferência entre Solanum americanum e o tomateiro. Aos resultados da produção de cinco ensaios, aplicaram-se modelos múltiplos de regressão, tendo como variáveis independentes a densidade e o número de dias de convivência entre a infestante e a cultura. A aplicação do Nivel de Tolerância (NT) à representação gráfica num plano das equações estimadas a partir de modelos múltiplos permite responder à questão: Quanto tempo pode permanecer uma determinada infestação de Solanum americanum sem causar prejuizo na cultura do tomateiro? É ainda possivel verificar que a variação dos custos do controle e dos preços da cultura faz variar o NT e, concomitantemente, o tempo admissivel, sem causar prejuizo, do Solanum americanum na cultura do tomateiro.
\end{abstract}

Palavras-chave: modelo múltiplo de competição, nível de tolerância, interferência.

\begin{abstract}
This study introduces a unifying concept combining the theory of critical density and critical period of weed infestation. It is hypothesized that these two periods are interdependent, rather than fixed values. The hypothesis is based on studies of interference between Solanum americanum and the tomato crop. Multiple regression models were applied to the results of five field trials. The independent variables were density and number of days of weed and culture cohabitation. Applying the Tolerance Level (TL) to the graphic representation (2D) of the equations estimated from multiple non-linear models allowed answering the following question: how long does a particular infestation of Solanum americanum remain without causing injury to the tomato crop? It was also possible to conclude that the variation of control costs and crop prices changes the TL values, concomitantly changing the time allowable for the presence of Solanum americanum in the tomato crop, without causing yield loss.
\end{abstract}

Keywords: multiple competition model,tolerance level, weed interference.

\section{INTRODUÇÃO}

Têm-se realizado nos anos mais recentes inúmeros trabalhos na área da interferência entre culturas e infestantes, podendo-se, de forma simplificada, dividir esses estudos em dois grandes grupos: trabalhos que avaliam a capacidade competitiva de cultivares de culturas em relação às infestantes (Dhima et al., 2000; Teixeira et al., 2009; Rigoli et al., 2009) e trabalhos que determinam os periodos críticos de interferência (Adkins et al., 2010;

1 Recebido para publicação em 20.6.2011 e aprovado em 15.9.2011.

2 Eng-ogro ${ }^{0}$, Dr., Professor, Instituto Politécnico de Beja, <http://www.esab.ipbeja.pt/>, Rua Pedro Soares 7800 - 295 Beja, Portugal, <jportugal@ipbeja.pt>; ${ }^{3}$ Eng-0-Agr ${ }^{\circ}$., Dr., Professor, Instituto Superior de Agronomia, Tapada da Ajuda 1349-017 Lisboa, Portugal (in memorian)

Planta Daninha, Viçosa-MG, v. 29, n. 4, p. 751-760, 2011 
Chauhan \& Johnson, 2010; Swanton et al., 2010).

Em ambos os tipos de estudo é frequente usar modelos matemáticos a fim de determinar o impacto da(s) infestante(s) na cultura, normalmente em nível da produção (Streibig et al., 2006; Vidal et al., 2010). Os modelos matemáticos usados nesses estudos podem ser de vários tipos, sendo comumente usados modelos do tipo linear e do tipo não linear hiperbólico. Portugal \& Moreira (2009) utilizaram esses dois tipos de modelo para determinação de niveis econômicos de prejuízos para estudar a interação entre Solanum americanum, conhecida por maria-pretinha, $\mathrm{e}$ a cultura do tomate. As variáveis explicativas para as perdas de produção nesse estudo foram a densidade e os períodos de competição. Contudo, esses modelos foram aplicados utilizando-se uma variável explicativa (a densidade), sendo aplicados individualmente a cada periodo de competição.

Esses tipos de modelo, aplicados de forma estanque para cada período de competição, fornecem informação importante quanto ao impacto da infestante para cada densidade e período de tempo ensaiados, porém acabam por dar sempre uma visão limitada do conjunto das duas variáveis avaliadas. Além disso, o ajuste dos modelos às variáveis, embora fosse bom, pode ainda ser melhorado com a utilização de modelos múltiplos, ou seja, que façam uso de mais variáveis. Convém mencionar que a escolha das variáveis para integrar esses modelos deve ser criteriosa e em reduzido número, pois, de outro modo, eles deixam de ser práticos. Assim, é desejável que as variáveis escolhidas estejam bem relacionadas com o efeito que se pretende estudar. Por outro lado, as variáveis dependentes deverão ser facilmente obtidas por parte do(s) utilizador(es), de forma a serem efetivamente úteis.

Convém ressaltar, em favor dos modelos múltiplos, que eles têm grau de aderência aos dados dos ensaios similar ao dos modelos que utilizam a biomassa (massa fresca ou seca), como o demonstrou Fernandes (2003). Esse aspecto é tanto mais significativo quando se considera que a biomassa é uma variável muito bem relacionada com a variável dependente a avaliar (perdas de produção, por exemplo, ou outra relacionada com planta cultivada).
Dessa forma, essa variável é muito usada em estudos de interferência em múltiplas combinações cultura/infestante(s), como se comprova pelos estudos em batata-doce (Stephen et al., 2010; Meyers et al., 2010), em arroz (Bhagirath et al., 2010), em beterraba (Odero et al., 2010a,b) e em soja (Kelly \& Smoot, 2010).

A biomassa, todavia, é uma variável cuja determinação é mais exigente que a densidade e o número de dias de presença comum da cultura com a(s) infestante(s). Desse modo, há vantagem prática na utilização dos modelos múltiplos que usam essas variáveis explicativas. Este trabalho teve como principal objetivo aplicar dois modelos do tipo múltiplo, que utilizam simultaneamente as variáveis densidade de infestação de $S$. americanum e período de convivência, aos dados de produção de tomateiro de indústria. Paralelamente, será determinado o nível de tolerância da cultura à infestação de S. americanum, por meio da representação gráfica daqueles modelos sobre um plano. Finalmente, esse tipo de abordagem permite acrescentar aos conceitos de densidade crítica e período crítico de interferência um novo conceito, que conjuga os dois anteriores e responde à questão: Quanto tempo pode permanecer uma dada densidade de infestação sem causar prejuízo à cultura?

\section{MATERIAL E MÉTODOS}

Este artigo utiliza os resultados de cinco ensaios cuja localização, características físicas e químicas do solo, delineamento experimental adotado (modalidades e repetições), modo de implantação e condução dos ensaios se encontram descritos em Portugal \& Moreira (2009). Findo o ciclo da cultura, efetuou-se a colheita dos frutos e procedeu-se à sua pesagem, convertendo-se os dados em valores equivalentes a $\mathrm{kg} \mathrm{ha}^{-1}$.

\section{Modelos e análise dos dados}

Os modelos de competição para a determinação da relação entre a redução de produção da cultura com a densidade de infestação e com o tempo de competição são os seguintes:

$$
Y=\mathrm{const}+b d i+c t+g d i t
$$

\section{西}

e 


$$
Y=\text { const }+b d i+c d i^{2}+d t+e t^{2}+g d i t
$$

Na construção dos modelos consideraramse as perdas de produção $(Y)$ como variável dependente. Como variáveis independentes, foram considerados: a densidade (di; dens), a densidade ao quadrado $\left(d i^{2} ;\right.$ dens $\left.^{2}\right)$, o número de dias de competição, correspondentes a cada período $(t$; dias), o número de dias de competição ao quadrado $\left(t^{2} ;\right.$ dias $\left.^{2}\right)$ e a combinação da densidade e do número de dias de competição (dit; dens x dias). Os coeficientes $b, c, d, e, g$ correspondem aos parâmetros da equação. Para simplificar, o primeiro modelo (1) passa a ser designado de múltiplo linear, e o segundo (2), de múltiplo não linear. Convém ressaltar que se utilizaram os valores médios das perdas de produção, de forma a evitar "ruído" provocado pela dispersão dos resultados.

A comparação das equações e dos modelos fez-se com base nos valores do coeficiente de determinação nos modelos múltiplos, que é o $\mathrm{r}^{2}$ ajustado. Essa opção justifica-se pelo fato de o $\mathrm{r}^{2}$ ajustado deduzir o efeito positivo causado quando se junta mais uma variável ao modelo. Os modelos foram considerados tanto melhor adaptados quanto maior o valor do coeficiente de determinação. Em relação a esse parâmetro, são comentados os resultados obtidos nas equações estimadas.

Aos modelos linear e não linear também foram aplicados o teste de $\mathrm{t}$ (Student), aos parâmetros das equações, e o teste de F, às equações estimadas. O primeiro, com a intenção de conhecer o grau de associação entre os parâmetros das equações e as variáveis estudadas (densidade, dias, densidade ${ }^{2}$, dias ${ }^{2}$, densidade $\mathrm{x}$ dias). $\mathrm{O}$ segundo, para se conhecer a probabilidade de esses parâmetros serem todos simultaneamente zero e, assim, saber se as equações podem, ou não, ser usadas no cálculo de prejuízos. Para níveis de significância superiores a $5 \%$ de probabilidade do erro experimental, os efeitos das varáveis e dos modelos foram considerados não significativos, o que significa que acima desse valor, no caso do teste de $F$, as equações não foram consideradas passiveis de serem usadas.

O tratamento estatístico foi realizado com o programa SPSS (Statistical Package for Social Sciences) versão 11.0. Para a representação gráfica das equações, recorreu-se ao programa Statistica versão 5.
Conforme referido por Portugal \& Vidal (2009), a determinação do Nivel Prejudicial de Ataque (NPA), quando feita através dos modelos múltiplos, linear e não linear, utiliza a mesma fórmula, a qual tem como lacuna não fazer uso de toda a informação contida nas equações estimadas, nomeadamente no que se refere ao tempo de competição. Para evitar essa limitação e, simultaneamente, tirar maior partido dos modelos múltiplos usados neste estudo, propõe-se a adoção da seguinte equação

$$
Y^{*}=\frac{C}{P Y p p E} \times 100
$$

sendo $Y^{*}$ a redução de produção tolerável (\%); $C$, o custo dos meios de controle; $P$, o preço unitário da produção; $Y p p$, a produção potencial da cultura isenta de infestantes; e $E$, a eficácia dos meios de controle (varia entre 0 e 1 , correspondendo de 1 a $100 \%$ ).

Utilizando a equação anterior, determinase a redução de produção tolerável, sendo o valor obtido uma porcentagem $\left(Y^{*}\right)$, a partir da qual é economicamente vantajoso combater a infestante. Esse valor, aplicado aos gráficos de "curvas de nivel" construídos a partir dos modelos múltiplos de competição, permite conhecer o período de tempo (número de dias) que uma determinada densidade da infestante pode permanecer na cultura sem causar prejuízos superiores ao custo do seu controle.

No cálculo dos niveis de tolerância, recorreu-se a valores correntes nos anos em que foram efetuados os ensaios, assim como a valores correntes do ano passado (2010), que são apresentados na Tabela 1.

\section{RESULTADOS E DISCUSSÃO}

Os valores dos parâmetros das equações de regressão estimadas para os modelos múltiplos linear e não linear e também os resultados da aplicação do teste de $t$ aos parâmetros e do teste $\mathrm{F}$ aos modelos de regressão (Tabela 2) permitem comparar e contrastar os diferentes ensaios.

No primeiro ensaio, no modelo linear, apenas o nivel de significância dos valores de t relativos ao parâmetro associado à interação dens $\mathrm{x}$ dias mostrou-se significativo. Assim, apenas a ação combinada da densidade e dos 
dias de competição afetou as perdas de produção do tomateiro. No modelo não linear, o parâmetro associado à dens ${ }^{2}$ mostrou-se significativo, sendo os valores de constante, dias, $\operatorname{dias}^{2}$ e dens x dias muito significativos. Esses resultados, por sua vez, indicam que o número de dias de competição, o efeito combinado das duas variáveis e a densidade em menor grau, na forma como a regressão se traduz, contribuem significativamente para as perdas de produção. Ainda assim, ambas as equações podem ser usadas com idêntico grau de segurança, atendendo à significância de F (Tabela 2).

Nos outros dois ensaios em sementeira direta (quarto e quinto), também se verificaram níveis de significância nos parâmetros testados (Tabela 2). No quarto ensaio, foram significativos os parâmetros associados às variáveis dens e dens $\mathrm{x}$ dias no modelo linear e dens, dens ${ }^{2}$ e dens $\mathrm{x}$ dias no não linear, o que indica que a densidade e a interação da densidade com o número de dias de competição estão relacionadas com as perdas de produção, ainda que a densidade esteja menos influente do que a interação. No quinto ensaio, no modelo não linear, não foi verificada significância para a variável densidade, mas apenas para a variável tempo (dias e dias ${ }^{2}$ ) e a interação dens $\mathrm{x}$ dias. Com base no nível de significância do teste de $\mathrm{F}$, verificou-se que ambas as equações podem ser usadas, uma vez que a hipótese de os parâmetros das variáveis serem simultaneamente zero é altamente improvável (Tabela 2).

Nos ensaios com raiz nua (segundo ensaio) e protegida (terceiro ensaio), as significâncias foram menores. No segundo ensaio, apenas a ação dos dias de competição exerceu atividade com significado nas perdas de produção, de acordo com a equação do modelo linear. Na outra equação, nenhum dos parâmetros associados às variáveis testadas mostrou ligação significativa. Contudo, qualquer das equações pode ser usada no cálculo de prejuízos, tendo em consideração o nível de significância do teste F. No terceiro ensaio, apesar de

Tabela 1 - Fatores e valores adotados nos cálculos dos níveis de tolerância nos ensaios de interferência entre tomateiro e mariapretinha. Instituto Politécnico de Beja, Portugal, 2011

\begin{tabular}{|c|c|c|c|c|c|c|}
\hline Fator $\quad$ Ensaio/ano & $1^{\circ}$ Ensaio & $2^{\circ}$ Ensaio & $3^{-}$Ensaio & $4^{\circ}$ Ensaio & $5^{\circ}$ Ensaio & 2010 \\
\hline Preço da monda $\left(\mathrm{R} \$ \mathrm{ha}^{-1}\right)$ & 400 & 450 & 820 & 870 & 910 & 1.000 \\
\hline Produção paga $(\mathrm{kg})$ & 80.351 & 58.641 & 93.519 & 66.923 & 89.643 & - \\
\hline Preço tomate $\left(\mathrm{R} \$ \mathrm{~kg}^{-1}\right)$ & 0,1981 & 0,2077 & 0,209 & 0,2009 & 0,1867 & 0,1867 \\
\hline
\end{tabular}

Taxa de câmbio $\cdot 1,00=\mathrm{R} \$ 2,28$.

Tabela 2 - Parâmetros associados aos modelos múltiplos linear ${ }^{1 /}$ e não linear²/ e às perdas de produção em função da densidade e do período de interferência entre tomateiro e maria-pretinha. Instituto Politécnico de Beja, Portugal, 2011

\begin{tabular}{|c|c|c|c|c|c|c|c|c|c|}
\hline Ensaio & $\begin{array}{c}\text { Perda } \\
\text { produção (\%) } \\
\end{array}$ & Const. & Dens & Dens $^{2}$ & Dias & Dias $^{2}$ & Dens x Dias & $r^{2}$ aj & $\mathrm{F}$ \\
\hline \multirow{2}{*}{$1^{\underline{o}}$} & $\mathrm{Y}$ & $-12,264$ & $-0,731$ & - & 0,246 & - & $0,119^{*}$ & 0,819 & $23,580^{* * *}$ \\
\hline & $\mathrm{Y}$ & $-99,336^{* *}$ & 5,561 & $-1,024^{*}$ & $2,645^{* *}$ & $-0,015^{* *}$ & 0,119 ** & 0,923 & $37,067 * * *$ \\
\hline \multirow{2}{*}{$2^{\underline{o}}$} & $\mathrm{Y}$ & $-12,457$ & 2,419 & - & $0,188^{*}$ & - & 0,013 & 0,795 & $25,524 * * *$ \\
\hline & $\mathrm{Y}$ & $-23,802$ & 3,175 & $-0,123$ & 0,488 & $-0,002$ & 0,013 & 0,774 & $13,992 * * *$ \\
\hline \multirow{2}{*}{$3^{o}$} & $\mathrm{Y}$ & $-3,692$ & 5,670 & - & 0,016 & - & 0,031 & 0,815 & $17,163 * *$ \\
\hline & $\mathrm{Y}$ & $-17,039$ & $-0,387$ & 2,019 & 0,608 & $-0,005$ & 0,031 & 0,866 & $15,200^{* *}$ \\
\hline \multirow{2}{*}{$4^{\circ}$} & $\mathrm{Y}$ & 2,127 & $-15,543^{*}$ & - & $-0,060$ & - & $0,444 * *$ & 0,870 & $34,367 * * *$ \\
\hline & $\mathrm{Y}$ & $-21,032$ & $-27,890 * *$ & $8,231 *$ & 0,664 & $-0,005$ & $0,444 * * *$ & 0,912 & $31,920 * * *$ \\
\hline \multirow{2}{*}{$5^{\circ}$} & $\mathrm{Y}$ & $-3,113$ & 5,790 & - & 0,042 & - & $0,001^{*}$ & 0,828 & $25,046^{* * *}$ \\
\hline & $\mathrm{Y}$ & $-42,308$ & $-9,183$ & 2,301 & $1,172 * *$ & $-0,007 * *$ & $0,277^{* * *}$ & 0,923 & $36,911 * * *$ \\
\hline
\end{tabular}

${ }^{1 /} \mathrm{Y}=\mathrm{const}+$ bdi $+\mathrm{ct}+$ gdit. ${ }^{2} / \mathrm{Y}=\mathrm{const}+\mathrm{bdi}+\mathrm{cdi}^{2}+\mathrm{dt}+\mathrm{et}^{2}+$ gdit. Y- perdas de produção (\%); const- constante; dens - densidade infestante; $\mathrm{t}$ - dias competição; b, c, d, e, g - parâmetros da equação. $\mathrm{P}$ - significância $* 0,01<\mathrm{P}<0,05 ; * * 0,001<\mathrm{P}<0,001 ; * * * \mathrm{P}<0,001$. 
nenhum dos parâmetros associados às variáveis das equações se mostrar significativo, as duas equações podem ser usadas nos cálculos de prejuizos, atendendo aos valores da significância do teste F (Tabela 2).

De acordo com os valores dos coeficientes de determinação, porcentagens superiores a 80 e $90 \%$ da variação das perdas de produção são explicadas, respectivamente, pelo modelo múltiplo linear e múltiplo não linear em todos os ensaios, exceto no terceiro, em que aqueles valores foram numericamente menores (79 e $77 \%$, respectivamente). Os coeficientes de determinação estimados são, em todos os casos, superiores aos determinados por Portugal \& Moreira (2009), quando se aplicaram aos ensaios os modelos linear e não linear (hiperbólico).

Convém ressaltar que as equações devem ser usadas apenas no intervalo dos valores testados (densidade e número de dias), fato que se considerou para elaborar a Figura 1. Ou seja, as densidades testadas nos diversos experimentos se situaram entre 0 e 6 plantas $\mathrm{m}^{-2}$. Em termos práticos, isso significa que sob níveis de densidade superiores a esses valores não há dúvida da necessidade de controle da infestante.

$\mathrm{Na}$ hipótese de se considerarem valores de densidade e de dias próximos de zero, ou mesmo iguais a zero, os modelos indicaram aumento ou diminuição de produção conforme o valor da constante (Tabela 2), que no caso do modelo múltiplo não linear, no primeiro ensaio, corresponderia a um aumento de produção ao redor de $100 \%$, o que é inverossimel.

As superficies de resposta do modelo múltiplo não linear aplicado nos cinco ensaios são apresentadas na Figura 1, na forma de três dimensões e com "curvas de nível".

O modelo múltiplo não linear, com exceção do segundo ensaio, é o que melhor se ajustou aos dados dos ensaios, uma vez que o valor do seu coeficiente de determinação ( $\mathrm{r}^{2}$ aj.) foi numericamente superior ao do coeficiente de determinação do modelo simples (Tabela 2). No entanto, o valor da significância de F não foi diferente para ambos os modelos (Tabela 2). Dessa forma, decidiu-se apresentar a representação gráfica (Figura 1), bem como o nível de tolerância (Figura 2), apenas para o modelo múltiplo não linear.

Em todos os ensaios, houve relação direta entre a densidade da infestante a a perda do rendimento do tomateiro (Figura 1). Todavia, em relação ao número de dias de competição, verificaram-se diferenças no seu efeito nas perdas, nos cinco ensaios. Assim, no primeiro ensaio, observa-se que, à medida que aumentou o número de dias de competição, ocorreu incremento na quebra de produção. O mesmo fenômeno pode ser observado no quarto e quinto ensaios, a partir de 0,5 planta de mariapretinha por metro quadrado. Nos ensaios transplantados, em especial no terceiro (raiz protegida), verificou-se que o período de competição condiciona menos as perdas de produção que nos ensaios feitos em sementeira direta (primeiro, quarto e quinto ensaios) (Figura 1).

Outro aspecto que merece destaque é que a expressão da variável número de dias depende das densidades testadas. De fato, verifica-se que em condições de densidades elevadas ocorre maior expressão do número de dias nas perdas (Figura 1).

Na Tabela 3 são apresentados os níveis de tolerância calculados, tendo-se adotado na sua determinação a equação apresentada anteriormente e os valores constantes na Tabela 1 . Considerou-se nos cálculos que a eficácia da monda se situa nos $85 \%$.

Os niveis de tolerância determinados nos cinco ensaios variaram de 2,9 a 7,6 plantas $\mathrm{m}^{-2}$ para as simulações realizadas com valoresbase (Tabela 1). Todavia, a faixa de NT foi alterada para 6,8 a 10,8 plantas $\mathrm{m}^{-2}$ quando se consideraram valores monetários de 2010 (Tabela 1).

O percentual de perda de produção necessária para compensar economicamente a realização do controle da infestante aumentou todos os anos até o quarto ensaio, a preços praticados nos anos dos ensaios (Tabela 3). $O$ resultado deve-se fundamentalmente ao sucessivo aumento do preço da medida de controle utilizada. Com efeito, no período de tempo compreendido entre o primeiro e o quarto ensaio (Tabela 3), o custo do controle teve aumento de $117 \%$. 

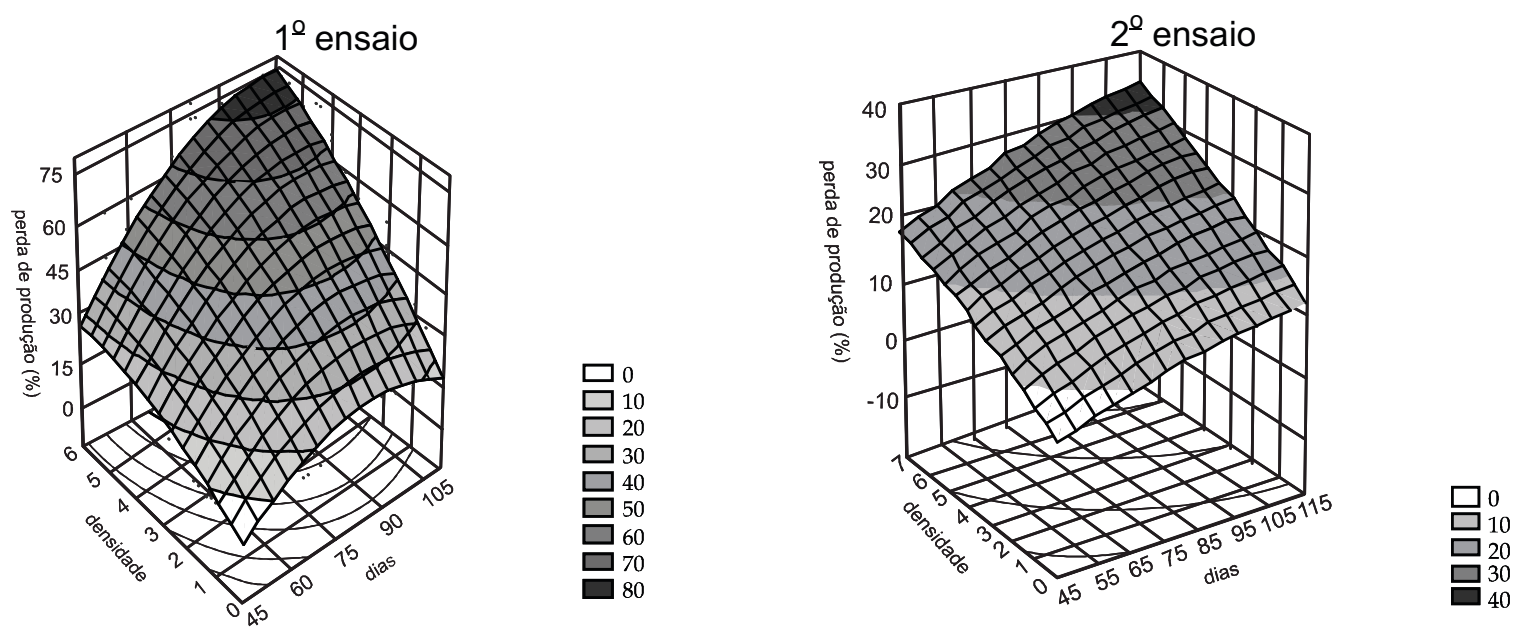

$\mathrm{Y}=-99.336+5.561 \mathrm{di}-1.024 \mathrm{di}^{2}+2.645 \mathrm{t}-0.015 \mathrm{t}^{2}+0.119 \mathrm{dit}$

$Y=-23.802+3.175 d i-0.123 d i^{2}+0.488 t-0.002 t^{2}+0.013 d i t$
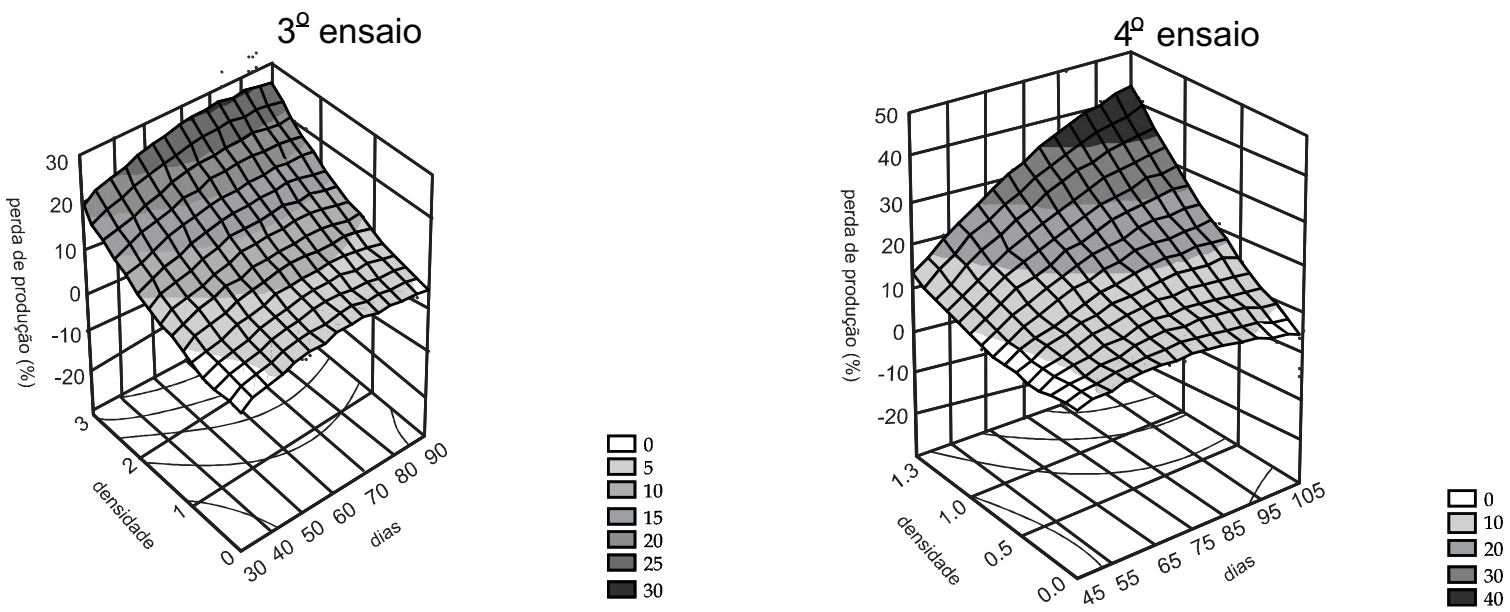

$Y=-17.039-0.387 d i+2.019 d i d^{2}+0.608 t-0.005 t^{2}+0.866 d i t$

$Y=-21.032-27.89 d i+8.231 d^{2}+0.664 t-0.005 t^{2}+0.444 d i t$

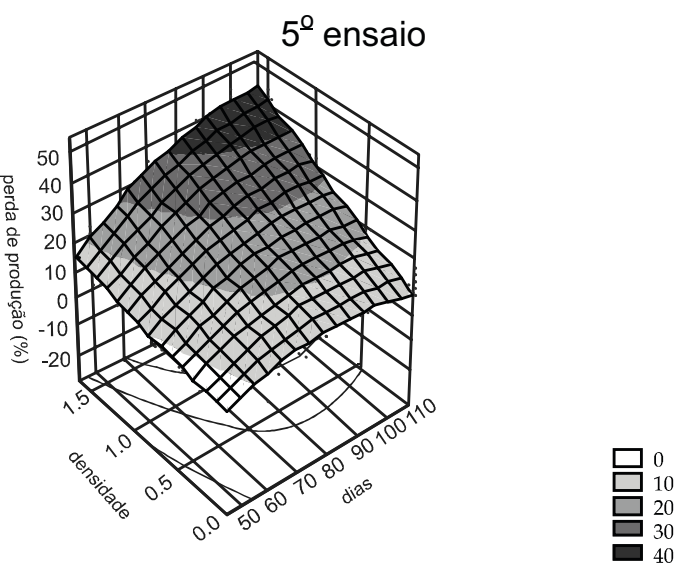

$Y=-42.308-9.183 d i+2.301 d i^{2}+1.172 t-0.007 t^{2}+0.277 d i t$

Figura 1 - Representação das equações ajustadas do modelo múltiplo não linear às perdas de produção do tomateiro, em função da densidade de Solanum americanum e do período de convivência entre com tomateiros. 

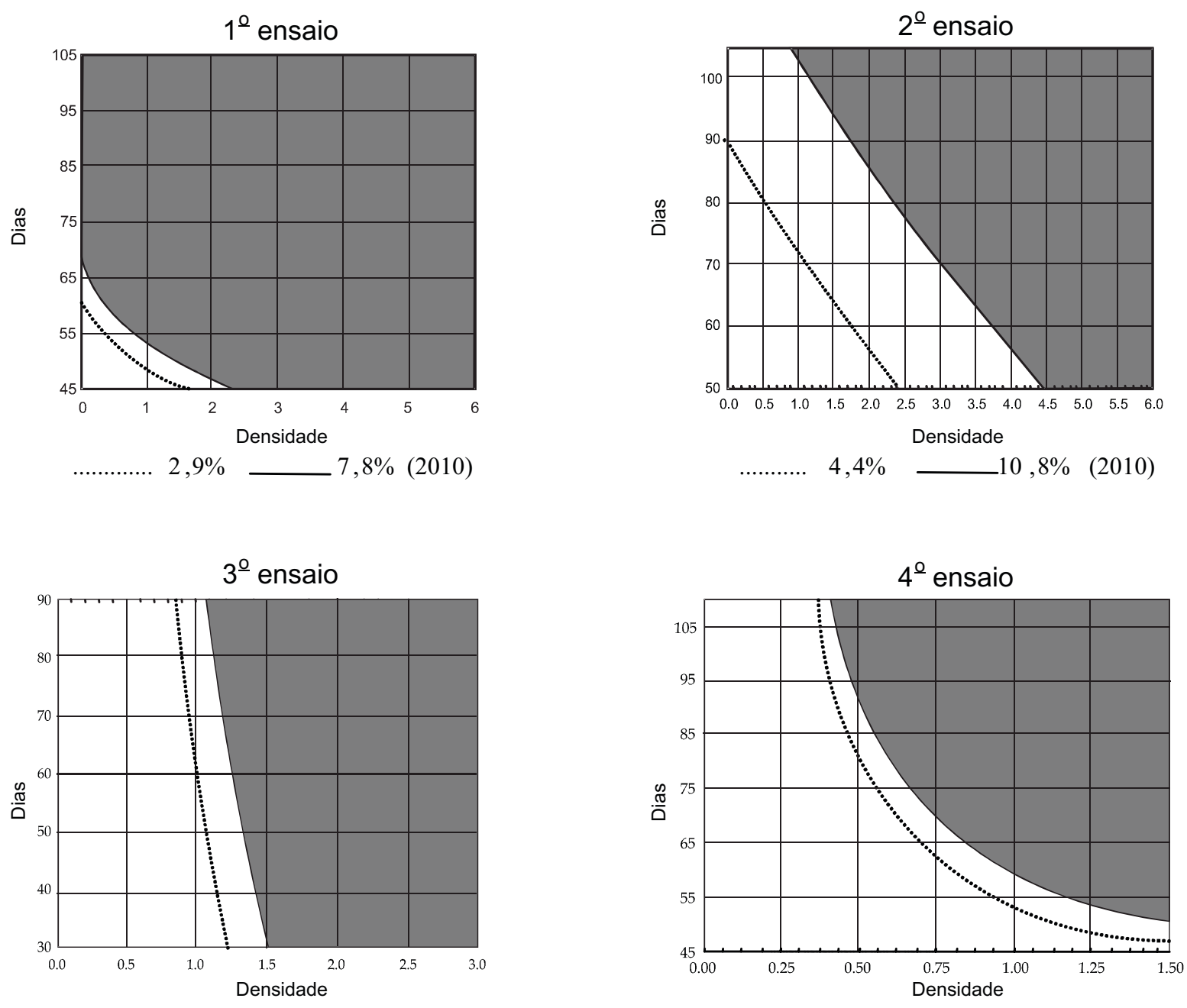

$4,9 \%-6,8 \%(2010)$

o

. $.7 .6 \%$

$9,4 \%(2010)$

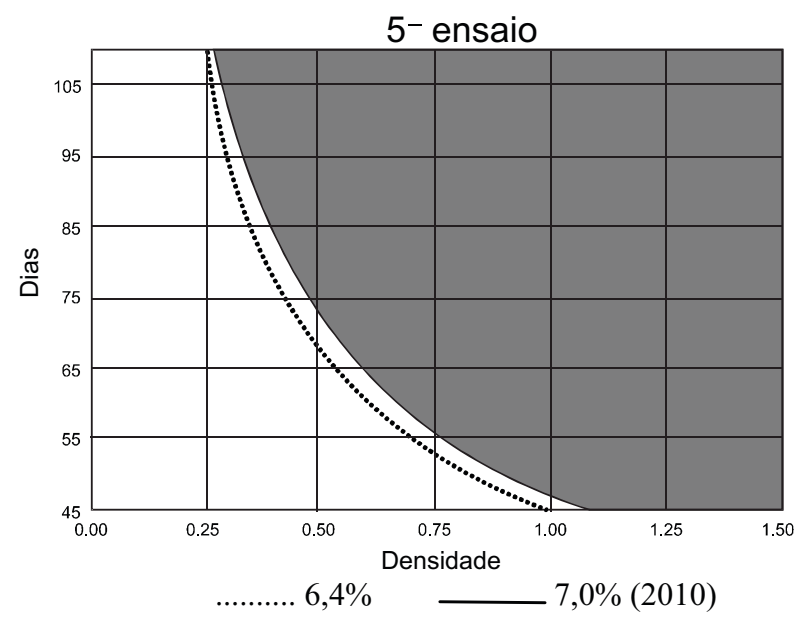

Figura 2 - Níveis de tolerância calculados com custos e preços no ano em que se efetuou o ensaio (linha ponteada) e em 2010 (linha cheia). 
No que se refere aos NT estimados em 2010 , verificou-se variação entre o valor mais alto e o mais baixo de $4 \%$ (Tabela 3 ). Isso se deve exclusivamente à diferença de produtividade registrada nos ensaios, sugerindo, portanto, que o rendimento da cultura tem também peso importante nos niveis de prejuízo calculados.

As "curvas de nivel" (Figura 2) correspondem às equações dos modelos múltiplos mais adaptadas aos dados dos ensaios, respectivamente para o primeiro, segundo, terceiro, quarto e quinto ensaios. As duas linhas traçadas, ponteada e cheia, representam, respectivamente, os NT estimados com os preços do ano do ensaio e os NT a preços de 2010. Acima dessa segunda linha, a área cinza indica a condição em que é economicamente vantajoso controlar as plantas de $S$. americanum.

Os NT correspondem ao resultado da interação entre o número de dias considerado e as várias densidades da espécie infestante. Assim, no primeiro ensaio podem permanecer até o início da floração (45 dias) duas plantas $\mathrm{m}^{-2}$ de maria-pretinha (Figura 2). Esse valor reduz para cerca de 0,5 planta $\mathrm{m}^{-2}$ quando se atinge a floração ( \pm 60 dias) (Figura 2$)$.

O efeito da alteração dos preços foi particularmente visível no segundo ensaio (Figura 2). Verifica-se que o aumento do NT traduz-se em diferenças muito significativas no número de maria-pretinhas que podem permanecer na cultura sem causar prejuízo. Uma vez que as densidades foram iguais às do ensaio anterior, esse gráfico mostra ainda a importância do modo de implantação da cultura e da produtividade nos niveis de prejuizo. Recorde-se que neste ensaio a cultura foi transplantada com plantas de raiz nua, e no anterior, de sementeira direta. Além disso, a produtividade do tomateiro foi inferior no segundo em relação ao primeiro ensaio. De fato, pode permanecer até a colheita 0,9 planta $\mathrm{m}^{-2}$ de ervas-moiras (Figura 2).

Quando a simulação de NT foi realizada para o terceiro ensaio com valores monetários de 2010, evidencia-se que pode permanecer 1,1 planta $\mathrm{m}^{-2}$ de ervas-moiras até o fim do ciclo da cultura (Figura 2). Destaca-se que neste ensaio a produtividade foi elevada, provavelmente devido ao modo de implantação da cultura (transplantio de plantas de raiz protegida). Assim, esse modo de implantação permite aos tomateiros maior capacidade competitiva em relação às plantas de maria-pretinha, quando comparado a outras modalidades de implantação da cultura.

O comportamento observado no quarto e quinto ensaios é parecido, sendo, contudo, os niveis tolerados um pouco mais baixos no último ensaio. Tendo em consideração que o modo de implantação foi o mesmo, e que o delineamento experimental também foi o mesmo, a diminuição da densidade de mariapretinhas tolerada deve-se exclusivamente à maior produtividade da cultura, que se verificou no quinto ensaio.

Convém destacar que a aplicação dos NT na forma aqui apresentada, isto é, de forma gráfica num dos planos dos modelos múltiplos, representa uma desconstrução do conceito de que o período crítico e a densidade crítica são valores fixos e independentes. Isso porque o período crítico não é fixo, mas varia com a densidade de infestação (Figura 2). Além disso, pode-se concluir que não há uma densidade crítica fixa, uma vez que depende do período de competição (Portugal, 2010). Os aspectos agora mencionados representam um dos maiores benefícios da utilização dos modelos múltiplos. A Figura 2 ilustra essa hipótese, ainda que não a três dimensões, mas num

Tabela 3 - Níveis de tolerância (NT) estimados nos cinco ensaios de interferência entre tomateiro e maria-pretinha. Instituto Politécnico de Beja, Portugal, 2011

\begin{tabular}{|c|c|c|c|c|c|}
\hline Ano & $1^{\circ}$ Ensaio & $2^{\circ}$ Ensaio & $3^{\circ}$ Ensaio & $4^{\circ}$ Ensaio & $5^{\circ}$ Ensaio \\
\hline $\mathrm{NT}(\%)^{\frac{1 /}{}}$ & 2,9 & 4,4 & 4,9 & 7,6 & 6,4 \\
\hline NT $(\%)^{\frac{2 /}{1}}$ & 7,8 & 10,8 & 6,8 & 9,4 & 7,0 \\
\hline
\end{tabular}

${ }^{1 /}$ preços correntes no ano do ensaio. ${ }^{2 /}$ preços correntes em 2010. 
plano. Ao invés de considerar a densidade de infestantes e os periodos de convivência isoladamente e com valores fixos, evidencia-se que a densidade e o período crítico são mutuamente dependentes (Figura 2). Essa abordagem permite determinar durante quanto tempo se suporta, sem prejuizo, uma determinada densidade.

Em conclusão, os níveis de tolerância, quando aplicados em representações gráficas de modelos múltiplos, num plano, que tenham como parâmetros a densidade de infestação e os dias de convivência comum entre infestante(s) e cultura (Figura 2), permitem responder à questão: Quanto tempo pode permanecer uma determinada densidade de infestação sem causar prejuízo? Chama-se a atenção para o fato de que essa informação é muito superior às oriundas de duas perguntas isoladas: a) A partir de que densidade de infestação verificase redução de produção significativa (conceito de densidade crítica)?; e b) Em que fase(s) do ciclo da cultura a competição é significativa (conceito de período crítico)? Em face do exposto, ressalta-se a importância dos modelos múltiplos, pois formulam uma questão que integra as duas anteriores.

Este trabalho evidenciou que o conhecimento da densidade da espécie daninha e, simultaneamente, do periodo de convivência da infestante com a cultura permite estabelecer o nível de tolerância. Dessa forma, é possivel saber durante quanto tempo a presença da infestante é economicamente suportável na cultura. Essa informação possibilita estabelecer prioridades nas operações culturais a serem realizadas, uma vez que se conhece antecipadamente até quando se deve fazer o combate à infestante. Essa informação, contudo, em algumas situações, devido a limitações operacionais, poderá não ter aplicação prática, porém, do ponto de vista conceitual, pode-se considerar uma abordagem valiosa.

\section{AGRADECIMENTO}

Ao Prof. Ribas Antonio Vidal, pelas sugestões numa versão preliminar deste texto, mas sobretudo pelo ânimo que me deu para que este trabalho tivesse maior visibilidade.

\section{LITERATURA CITADA}

ADKINS, J. I. et al. Critical period of interference between American black nightshade and triploid watermelon. Weed Technology, v. 24, n. 3, p. 397-400, 2010.

BHAGIRATH, S.; CHAUHAN, B. S.; JOHNSON, D. E. Responses of rice flatsedge (Cyperus iria) and barnyardgrass (Echinochloa crus-galli) to rice interference. Weed Sci., v. 58, n. 3, p. 204-208, 2010.

DHIMA, K. V.; ELEFTHEROHORINOS, I. G.; VASILAKOGLOU, I. B. Interference between Avena sterilis, Phalaris minor and five barley cultivars. Weed Res., v. 40, n. 6, p. 549-559, 2000.

FERNANDES, J. M. P. V. Ecologia da flora espontânea e competição da erva-moira (Solanum nigrum L.) na cultura do tomate para indústria. 2003. 225 f. Tese (Doutorado em Agronomia)-Universidade Técnica de Lisboa, Lisboa, 2003.

KELLY A. N.; SMOOT R. L. Yellow Nutsedge (Cyperus esculentus) interference in soybean. Weed Technology, v. 24, n. 1, p. $39-43,2010$

MEYERS, S. L. et al. Interference of palmer amaranth (Amaranthus palmeri) in sweet potato. Weed Sci., v. 58, n. 3, p. 199-203, 2010.

ODERO, D. C. et al. Lanceleaf sage (Salvia reflexa) interference in sugarbeet. Weed Technology, v. 24, n. 4, p. $557-561,2010$ a.

ODERO, D.C. et al. Wild buckwheat (Polygonum convolvulus) interference in sugarbeet. Weed Technology, v. 24, n. 1, p. $59-63,2010$ b.

PORTUGAL, J. Nível crítico de dano (NCD) de infestantes na cultura do tomate de indústria. In: VIDAL, R.A.; PORTUGAL, J.; SHORA NETO, F. Nível crítico de dano de infestantes em culturas anuais. Porto Alegre: Evangraf, 2010 , p. $57-72$

PORTUGAL, J. M.; MOREIRA, I. Impacto da erva-moira na produção do tomateiro e ajuste de modelos matemáticos. Planta Daninha, p. 901-911, 2009. (Número Especial)

PORTUGAL, J. M.; VIDAL, R. A. Níveis econômicos de prejuízos de plantas infestantes nas culturas agrícolas: conceitos, definições e formas de cálculo. Planta Daninha, v. 27 , n. 4 , p. $869-877,2009$

RIGOLI, R.P. et al. Potencial competitivo de cultivares de trigo em função do tempo de emergência. Planta Daninha, v. 27, n. 1, p. $41-47,2009$.

SWANTON, C. J.; O'SULLIVAN, J.; ROBINSON, D. E. The critical weed-free period in carrot. Weed Sci., v. 58, n. 3, p. 229-233, 2010.

Planta Daninha, Viçosa-MG, v. 29, n. 4, p. 751-760, 2011 
STEPHEN L. et al. Interference of palmer amaranth (Amaranthus palmeri) in Sweetpotato. Weed Sci., v. 58, n. 3 , p. $199-203,2010$

STREIBIG, J. C. et al. Estimation of threshold for weed control in Australian cereals. Weed Res., v. 29, n. 2 , p. 117-126, 2006.
TEIXEIRA, I. R. et al. Competição entre feijoeiros e plantas daninhas em função do tipo de crescimento dos cultivares.

Planta Daninha, v. 27, n. 2, p. 235-240. 2009

VIDAL, R. A.; PORTUGAL, J.; SHORA NETO, F. Nível crítico de dano de infestantes em culturas anuais. Porto Alegre: Evangraf, 2010. 133 p. 